\title{
Model-Based Super-Resolution of SEM Images of Nano-Materials
}

Suhas Sreehari ${ }^{1}$, S. V. Venkatakrishnan ${ }^{2}$, Jeff Simmons ${ }^{3}$, Lawrence Drummy ${ }^{3}$, and Charles A. Bouman ${ }^{1}$.

${ }^{1 .}$ Electrical and Computer Engineering, Purdue University, West Lafayette, IN, USA.

2. Advanced Light Source, Lawrence Berkeley National Laboratory, Berkeley, CA, USA.

3. Air Force Research Laboratory, Dayton, OH, USA.

Many imaging problems in materials and biological sciences involve reconstruction of highresolution images from low-resolution SEM images that contain several similar or identical nonlocal structures. Such SEM images can be sparsely represented owing to the enormous redundancy caused by repeating structures. Model-based iterative reconstruction (MBIR) is a powerful iterative reconstruction framework that could theoretically exploit such redundancies [1]. However, in practice, determining a prior probability term in the maximum $a$ posteriori cost function that accounts for the similarity between non-local structures remains an open problem. Meanwhile, non-local patch-based denoising algorithms like non-local means (NLM) have been known to exploit non-local similarities in images. In fact, there have been several efforts to solve the super-resolution problem using patch-based models, dictionary learning and examplebased methods [2]. In any case, it is unclear how to use NLM-based denoising algorithms as prior models within the MBIR framework.

In this paper, we present a novel solution to the problem of super-resolution that works by using high-resolution patches from an external library. Our library-based non-local means (LB-NLM) algorithm contrasts the standard NLM in the quality and resolution of the patches that are weighted. The images interpolated to the desired higher resolution using LB-NLM are found to have lower mean squared error compared to linear methods like Shepard's interpolation, as well as solutions that use standard NLM. Additionally, the LB-NLM interpolated images are sharper, have textures visually consistent with ground truth images, and more accurate edge features. More importantly, our interpolated images lack resilient artifacts like jaggies that often manifest in super-resolution by factors around $4 \mathrm{x}$. The key step in our proposed solution is the use of LBNLM as a prior model in our model-based regularized inversion framework. This ordinarily challenging step is performed easily using the plug-and-play (P\&P) framework [3] that is based on the alternating direction method of multipliers (ADMM) [4]. The main benefit of the plugand-play framework is the decoupling of the forward and prior models of the MAP cost function. The forward model for this problem is given simply by the assumed point-spread function (PSF) of the electron microscope, while LB-NLM serves as the prior model.

In Figures 1 and 2, we present 4x super-resolution results on a scanning electron microscope image and a surface crack in the shell of marine mollusk, Hinea brasiliana. Along with the claims made above, we also show that a variety of denoising algorithms such as DSG-NLM [3] and library-based NLM (LB-NLM) can be plugged in as prior models using the P\&P framework. To implement the LB-NLM, we extracted 10,000 $5 \times 5$ patches from the "library images" (see Fig. 1, top left) to form the patch library. We did not add any noise to the 
measurements. The P\&P super-resolution algorithm with LB-NLM as the prior produced the best quality interpolated images with the lowest mean squared error, visually accurate textures and edge-features, and no jaggies. Our results demonstrate that using an external library of high quality patches within the flexible $\mathrm{P} \& \mathrm{P}$ framework can improve super-resolution quality much beyond traditional model-based as well as linear interpolation methods.

\section{References:}

[1] C. A. Bouman, "Model based image processing."

[2] S. C. Park, M. K. Park, and M. G. Kang, "Super-resolution image reconstruction: a technical overview," Signal Processing Magazine, IEEE, 20:3, pp. 21-36, 2003.

[3] S. Sreehari, S. Venkatakrishnan, B. Wohlberg, L. F. Drummy, J. P. Simmons, and C. A. Bouman, "Plug-and-play priors for bright field electron tomography and sparse interpolation," arXiv preprint arXiv:1512.07331, 2015.

[4] D. Gabay and B. Mercier, "A dual algorithm for the solution of nonlinear variational problems via finite element approxi- mation," Computers \& Mathematics with Applications, 2:1, pp. 17-40, 1976.

[5] This work was supported by AFOSR/MURI grant \#FA9550-12-1-0458, by UES Inc.

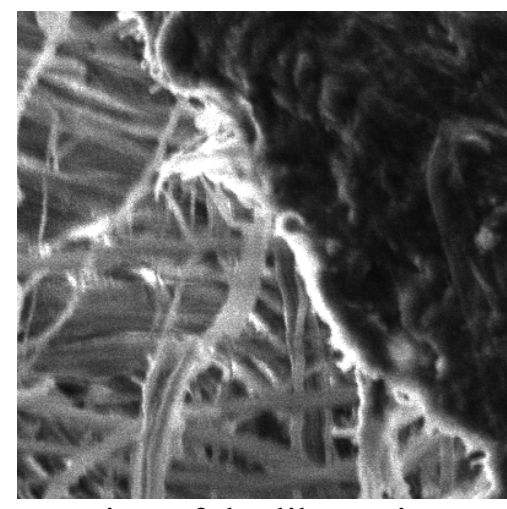

Portion of the library image

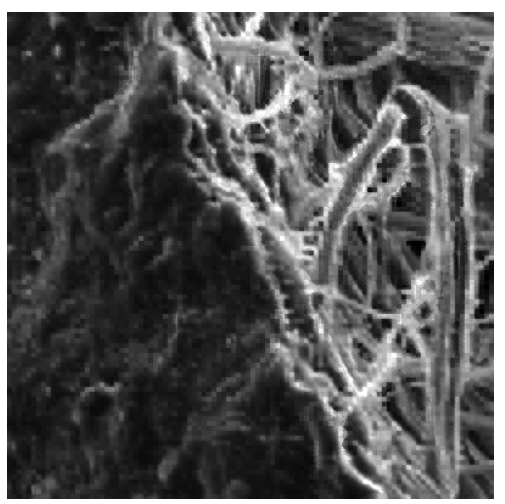

DSG-NLM interpolation

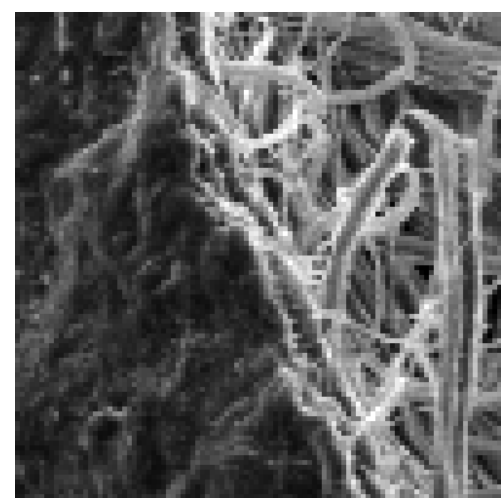

Low resolution image

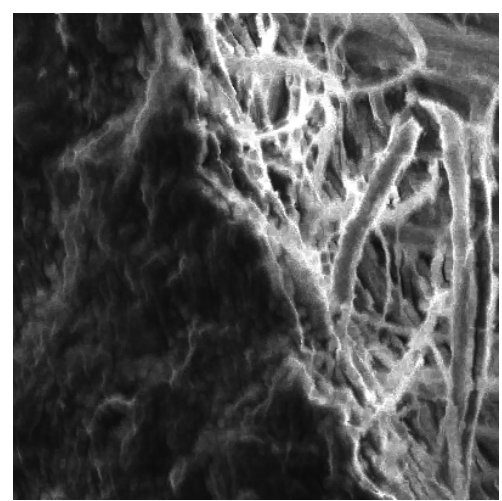

LB-NLM interpolation

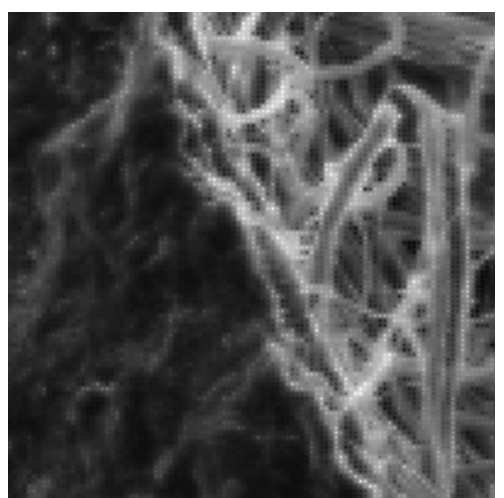

Shepard's interpolation

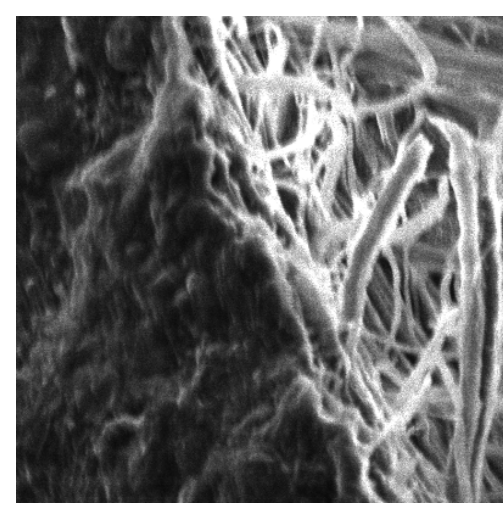

Ground truth

Figure 1. 4x super-resolution of a $100 \times 100$ SEM image of surface crack in the shell of the marine mollusk Hinea brasiliana. 